http://jmscr.igmpublication.org/home/ ISSN (e)-2347-176x ISSN (p) 2455-0450 crossref DOI: https://dx.doi.org/10.18535/jmscr/v8i6.36

\title{
Management of unstable Dorsal fracture - dislocation of the proximal interphalangeal joint by volar plate fixation with or without Bone graft
}

\author{
Authors \\ Dr Rajeev Kumar Rajak, M.S. (Ortho.), M.Ch. (Plastic) ${ }^{1}$, \\ Dr Arvind Kumar M.S. Ortho. ${ }^{2}$ \\ ${ }^{1}$ Assist. Professor, Dept. of Orthopedics, GMC, Bettiah \\ ${ }^{2}$ Professor and Head of the Dept. of Orthopedics, GMC, Bettiah
}

\begin{abstract}
Background: To evaluate the treatment of severe dorsal fracture dislocation (DFD) injuries of the proximal interphalangeal joint (PIPJ) by open reduction, bone grafting and fixation with mini-hook plates.
\end{abstract}

Method: Thirteen patients with extensive dorsal fracture dislocation of PIP joints were reconstructed with fabricated hook plate with elevation and bone grafting of depressed articular fragment.

Result: Hook plate treatment of PIP joint fracture dislocation restore articular anatomy and joint congruence at a single sitting and permits post operative mobilisation without the need for extension blocking splint.

Conclusion: Hook plate fixation is very useful in such circumstances and hook enables their ability to grasp the small volar lip fragment and early mobilization is possible.

Keyword: PIPJ, dorsal fracture dislocation, bone graft, mini hook plate.

\section{Introduction}

Fracture of base of middle phalanx involving the articular surface volar side are frequently associated with dorsal instability of PIP joint leading to dorsal subluxtion. Depressed articular fragment may co-exist with fracture of the volar lip, increasing both the extent of articular surface damage and degree of joint instability. Management of these complex injuries is challenging. Treatment broadly based on either directly reconstruct the volar lip of the middle phalanx through an open approach or re- establish joint congruence by closed means and indirectly allowing the volar lip fracture to re-modeled.
Accurate reduction of articular fragment by closed method cannot be always achieved. Direct volar lip reconstruction through open reduction and internal fixation technique by various ways have been described.

Small delicate fracture fragments are often difficult to reduce and hold with hard and hold with hardware and adjacent volar soft tissue and flexor tendons are susceptible to scarring with volar access incision. There is no clear consensus on the optimal treatment for such injuries. The present study open approach through volar incision fixing the fracture with volar buttress 
hook plate with bone graft for associated depressed articular fragment.

\section{Methods}

The patient admitted in GMC, Bettiah, Dept. of Orthopedics from October 2018 to April 2020 with unstable Dorsal fracture dislocation were included in this study. The patient with stable fracture dislocation of PIP joints were excluded.

The operative radiograpghy was used to quantify the estimation of percentage of the base of the middle phalanx articular surface involvement 25$50 \%, 50-60 \%$ and $60-75 \%$. Under GA / regional anesthesia and arm tourniquet PIP joint approached through a volar incision, elevate the flap of tendon sheath between distal A2 and proximal A4 pulley to allow the retraction of flexor tendon. Care was taken to preserve the attachment of volar plate to the fragment.

PIP joint was reduced. Dorsal blocking K- wire used to maintain the joint reduction. Depressed articular fragment was elevated and bone grafting done. A straight $1.3 \mathrm{~mm}$ LCP was cut and ends twisted to create hook plate, which was used to buttress the reconstructed volar lip. A single screw is sufficient to fix the plate adequately. $\mathrm{K}$ - wire is removed in fresh case but in older case remains for 3 weeks.

The hand was immobilized with volar slab and PIP joint in full extension for the first week. Exercise began after stitch removal.

\section{Results}

Total no. of pt. - 13 Men - 11, Women - 2 Right hand -9 Left hand- 4 Ring finger- 8 Little finger2 Middle finger- 2 Index finger- 1

8 case due to fall. One finger caught in machine. For case direct blow of heavy object for patient underwent for tendon repair. Range of movement flexion-90(70-110), extension $15\{(-10)$ to $(-35)\}$ arc of flexon-80(65-100).

\section{Discussion}

It has been demonstrated that a relatively large $\operatorname{ROM}\left(23^{\circ}-87^{\circ}\right)$ is necessary at the PIP joints for the functional use of the hand. Therefore restoration of motion is a key objective in treatment of Dorsal Fracture Dislocation after PIP injury, which in turn relies upon the joint congruity.

When considering articular congruence PIP joint stability against dorsal dislocation is dependent on the integrity the volar articular base of the middle phalanx. It is generally accepted that fracture disrupting greater part of the articular surface are more prone to subluxate. Tysere et al demonstrated that with $60 \%$ disruption of the middle phalanx volar lip, subluxation occurred at $67^{\circ}$ when moving towards extension.

Motion at the PIP joint during healing has been shown to have beneficial effects on cartilage remodeling even without perfect fracture reduction.

Inter fragmenting screw fixation for this fracture is not a reliable option because of cortex is very thin and communition is present.

So hook plate fixation is very useful in such circumstances and hook enables the ability to grasp the small volar lip fragment and early mobilization is possible.

\section{References}

1. NG CY, Oliver CW. Fractures of the proximal interphalangeal joints of the fingers. J Bone Surg Br. 2009;91(6):70512.

2. Newington DP, Davis TR, Barton NJ. The treatment of dorsal fracture- dislocation of the proximal interphalangeal joint by closed reduction and Krischner wire fixation: a 16- year follow up. J Hand Surg Br. 2001;26(6):537-40.

3. Tyser AR, Tsai MA, Parks BG, Means KR Jr. stability of acute dorsal fracture dislocations of the proximal interphalangeal joint: a biochemical study. J Hand Surg Am. 2014;39(1)13-8.

4. Weiss AP. Cerclage fixation for fracture dislocation of the proximal interphalangeal 
joint. Clin Orthop Relat Res. 1996(327): 21-8.

5. Bain GI, Polites N, Higgs BG, Hepstinstall $\mathrm{RJ}, \mathrm{McGrath}$ AM. The functional range of motion of the finger joints. J Hand Surg Eur Vol. 2015;40(4):406-11. 